\title{
Les agro-tensioactifs
}

\section{Jacky VANDEPUTTE}

IAR - Pôle de Compétitivité Industries et Agro-ressources, 50-52 rue Brossolette, BP 05, 02930 Laon cedex $<$ vandeputte@iar-pole.com>

\begin{abstract}
Agro-based surfactants
Thanks to their hydrophilic head and hydrophobic tail, surfactant molecules offers wetting, solubilizing, detergency and emulsifying properties, interposing themselves between water and water-insoluble substances. Main surfactant applications are washing/cleaning, and cosmetics; these sectors use more than $50 \%$ of the total amount of surfactants produced. In recent years a continuous trend towards surfactants based on renewable ressources, the agro-based surfactants, has become apparent. Here is an overview of the market, the regulation, the major renewable raw materials used and the agro-based surfactants developped by oleochemistry. It shows that the use of vegetable fats and oils allows the development of competitive, powerful products, which are both consumer-friendly and environment-friendly.
\end{abstract}

Key words: agro-based surfactants, market, regulation, renewable raw materials, properties

beauté) comme les crèmes, laits, maquillages et autres produits de soin (en tant qu'émulsionnant ou adoucissant). Ils entrent comme produits auxiliaires dans les procédés de fabrication de nombreux domaines industriels et agricoles (figure 1).

\section{Classification des tensioactifs}

Les tensioactifs sont classés suivant la nature de la tête polaire :

- anioniques (charge négative : carboxylates $\mathrm{COO}^{-}$, sulfonates $\mathrm{SO}_{3}{ }^{-}$, sulfates $\mathrm{OSO}_{3}^{-}$) ;

- cationiques (charge positive : amines $\mathrm{R}_{3} \mathrm{NH}^{+}$, ammoniums quaternaires $\mathrm{R}_{4} \mathrm{~N}^{+}$) ; - zwitterioniques (fonction anionique + cationique : bétaïnes, phospholipides) ;

- non ioniques (ethoxylats, polyols...).

Les composés non ioniques représentent $51 \%$ de la consommation en Europe, suivis des tensioactifs anioniques (40\%).

Les tensioactifs peuvent être classés en fonction de la partie lipophile (C8C10 : agents mouillants; C12-C16 : détergents ; $\mathrm{C} 18-\mathrm{C} 22$ : émulsifiants ou adoucissants) ou en fonction de leur HLB (hydrophilic/lipohilic balance) comme résumé figure 2.
Les applications industrielles et agricoles des tensioactifs

Les tensioactifs sont utilisés dans le traitement du cuir (préparation de la peau au tannage), la synthèse et formulation de matières plastiques, le nettoyage et dégraissage des matériaux, l'extraction de minerais, la formulation des peintures (stabilisation des formulations, mouillage des pigments...), les opérations de l'industrie pétrolière (du forage au rainage), la formulation de produits phytosanitaires et d'engrais (granulation, suspension des actifs phytosanitaires), le traitement du textile (ensimage, lubrification des fibres, opération de lavage et teinture).

\section{Le marché des agro-tensioactifs}

Les deux secteurs les plus consommateurs d'agro-tensioactifs sont la détergence ménagère et la cosmétique (figure 3 ) :

- la détergence ménagère (lessives, adoucissants, produits vaisselles et d'entretien) : 42000 tonnes, taux de pénétration de 20 à $25 \%$;

- la cosmétique : 35000 tonnes, taux de pénétration de 60 à $80 \%$;

Pour citer cet article : Vandeputte J. Les agro-tensioactifs. OCL 2012 ; 19(2) : 133-137. doi : 10.1684/ocl.2012.0431 


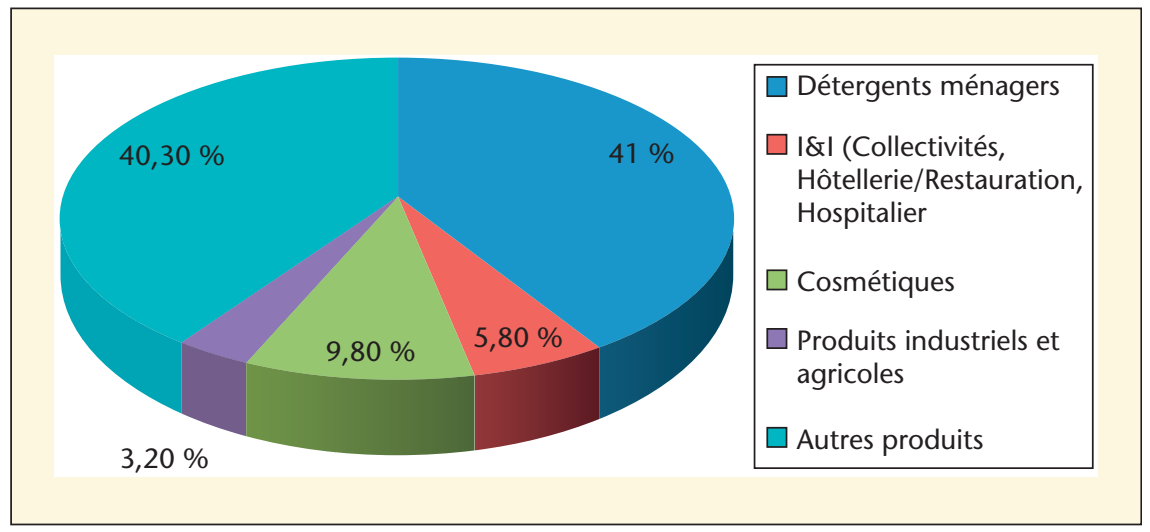

Figure 1. Segmentation du marché européen des tensioactifs selon leur application (données 2002).

- autres produits (cuir, forage, textile...) : 30000 tonnes, taux de pénétration de $15 \%$

- produits industriels et agricoles : 3000 tonnes, taux de pénétration de $20 \%$

- autres détergents : 2000 tonnes, taux de pénétration de $10 \%$.

\section{Comparatif de prix}

Le prix moyen d'un tensioactif est de 1 à 1,50 euros/kg (pour plus de $90 \%$ du marché). Dans le domaine de la détergence, il est compris entre 0,6 à 0,80 euro/kg. Sur certains marchés de niche, il peut atteindre 3 à 4 euros $/ \mathrm{kg}$ (figure 4).

\section{Prévisions du marché français en 2015}

La filière des tensioactifs est bien structurée. Elle compte une dizaine de producteurs de synthons rentrant dans la composition des agro-tensiactifs. La plupart des fabricants et distributeurs (plus de 120 en France) possèdent dans leur gamme une référence agro-tensioactive.

La production française est inférieure à la consommation.

\begin{tabular}{|c|c|}
\hline Zone HLB & Propriétés \\
\hline 4 à 6 & Émulsionnant E/H \\
\hline 7 à 9 & Agent mouillant \\
\hline 8 à 18 & Émulsionnant $\mathrm{H} / \mathrm{E}$ \\
\hline 13 à 15 & Détergent \\
\hline 10 à 18 & Solubilisant \\
\hline
\end{tabular}

Figure 2. Segmentation des domaines applicatifs en fonction de la HLB (hydrophilic/lipophilic balance).
Les experts tablent sur une croissance de 7,5\% sur les périodes 2005 à 2015 et 2015 à 2030.

\section{La réglementation}

\section{Le règlement $\mathrm{REACH}$}

Depuis le $1^{\text {er }}$ juin 2007, est entré en vigueur le règlement REACH (Registration, Evaluation, Authorisation and restriction of Chemicals) qui a pour but de remplacer les substances chimiques préoccupantes pour la santé et l'environnement. De nombreux tensioactifs sont concernés : les alkylphénols éthoxylés, les éthers sulfates, les alkylbenzènes sulfonates linéaires (LaBs).

\section{Le cadre réglementaire}

Depuis les années 1970, les fabricants de tensioactifs ont dû prendre en compte dans le développement de leurs nouveaux produits des critères liés principalement au devenir des tensioactifs dans l'environnement (toxicité, écotoxicité et biodégradabilité). Ainsi, la directive 73/ 404/ce (22/11/73) interdit la mise sur le marché de détergents si la biodégradabilité moyenne des tensioactifs est $<$ à $90 \%$; ; la directive 73/405/ce : impose aux tensioactifs anioniques d'avoir une biodégradabilité $>80 \%$; et la directive $82 / 242 / c e, 82 / 243 / c e, 86 / 94 / c e$ donne une méthode normée commune pour déterminer la biodégradabilité.

\section{Les matières premières renouvelables utilisées}

Les tensioactifs sont des molécules amphiphiles, constituées d'une partie hydrophile et d'une partie hydrophobe.

\section{Groupement hydrophile}

La tête polaire des agro-tensioactifs est constituée d'un carbohydrate ou d'une protéine. Elle peut être issue de coproduits de I'industrie de l'amidon ou de sucres : maltodextrine, glucose, saccharose. Elle peut être aussi du lactose, des polyols (sorbitol et xylitol), de l'inuline de chicorée, des pentoses (xylose et arabinose), du glycérol... Enfin, des oligopeptides et acides aminés issus du gluten de blé ou de maïs peuvent être utilisés. 


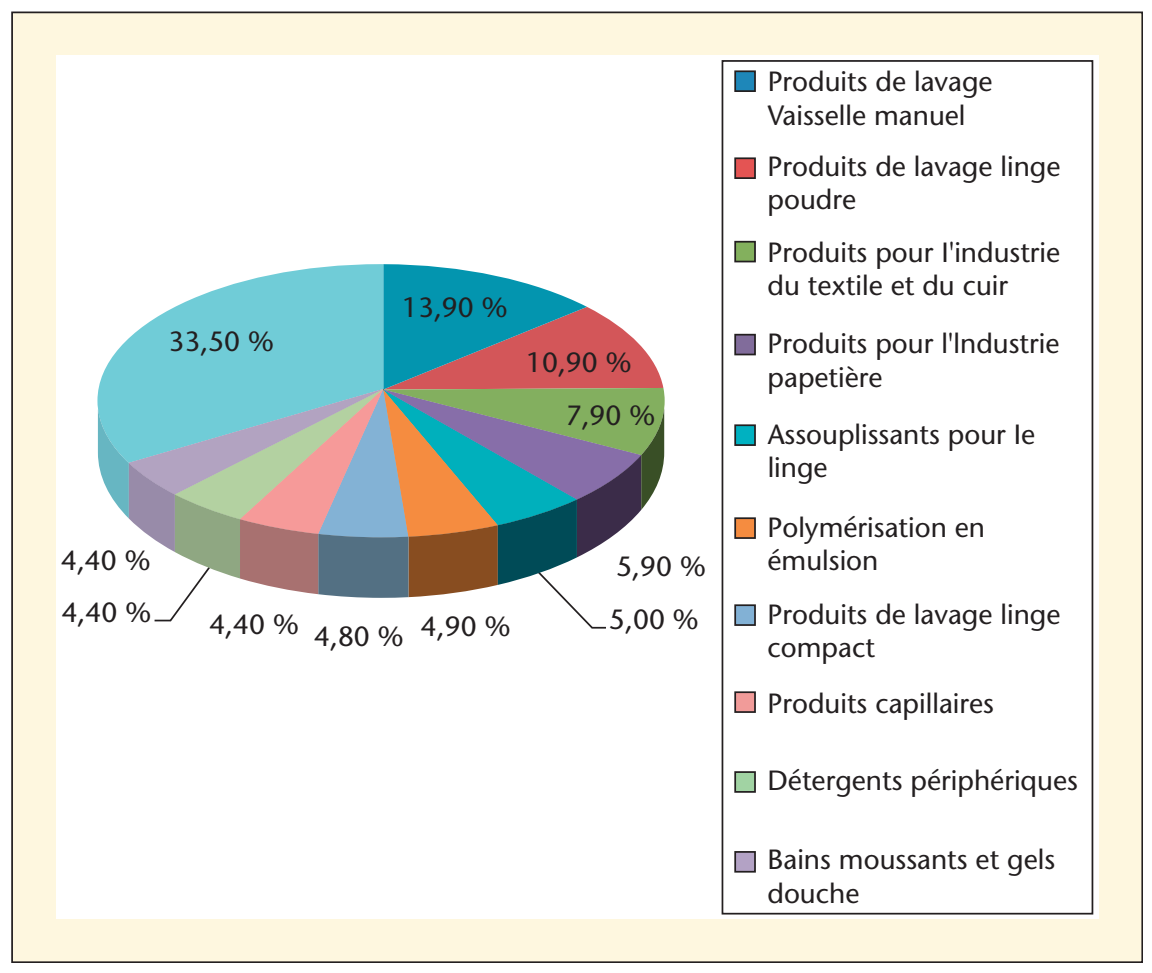

Figure 3. Segmentation fine du marché européen des tensioactifs selon leur application (données 2002).

\section{Groupement hydrophobe (= lipophile)}

La chaîne lipohile des agro-tensioactifs est essentiellement issue $d$ 'huiles végétales obtenues par trituration des graines des plantes oléagineuses: huile de coprah, huile de palme (palmefruit) -huile de palmiste. utilisés pour la synthèse de tensioactifs sont principalement les acides gras, les esters méthyliques, les amines grasses et les alcools gras. Les huiles sont constituées de triglycérides (figure 5). Leur hydrolyse conduit à la production d'acides gras ( $\mathrm{R}-\mathrm{COOH})$ et de glycérol qui peut servir pour le groupement hydro-
Les intermédiaires chimiques de base

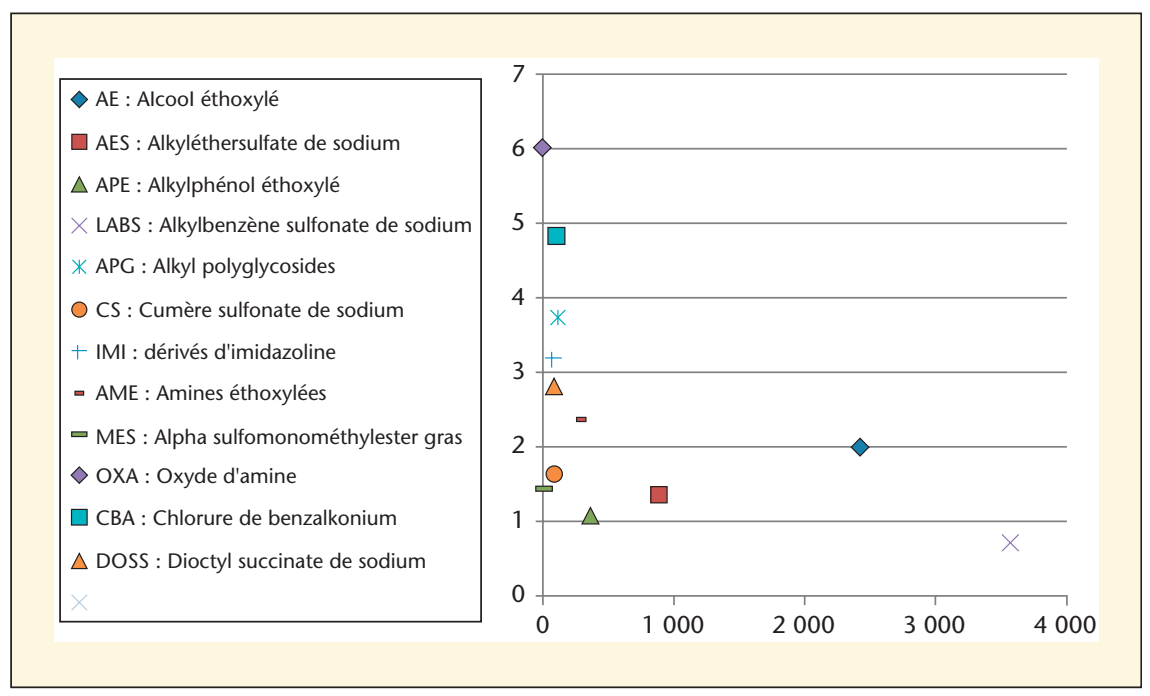

Figure 4. Prix des tensioactifs (euros $/ \mathrm{kg}$ ) en fonction des quantités mondiales consommées (milliers de tonnes). phile. Les principaux producteurs d'acides gras et d'esters méthyliques en Europe sont : Uniquema, Cognis, Oleon, Akzo Nobel ; les principaux producteurs $d^{\prime}$ alcool gras sont Cognis et Sasol; et les producteurs d'amines grasses sont $\mathrm{Ceca}$, Akzo Nobel et Clariant.

La variété des acides gras disponibles dans la nature est grande et est spécifique de la source considérée. Les acides gras majeurs sont les acides: laurique (C12), myristique (C14), palmitique (C16), stéarique (C18), oléique (C18:1), linoléique (C18:2), et linolénique (C18:3). À côté de ceux-ci, de nombreux acides gras sont utilisables. On utilisera certains acides gras originaux pour leurs particularités structurales (longueur de chaînes particulières, fonction chimique originale...).

Après le fractionnement des huiles, la distillation permet de séparer les acides gras en fonction de leur longueur de chaîne. Les esters méthyliques d'acides gras ( $\mathrm{R}-\mathrm{COOH} 3$ ) sont obtenus par transestérification des huiles avec du méthanol. Les alcools gras ( $\mathrm{R}-\mathrm{OH})$ sont fabriqués principalement à partir d'esters méthyliques $d^{\prime}$ acides gras. Les amines grasses ( $\mathrm{R}-\mathrm{NH} 2$ ) sont synthétisées essentiellement à partir $d^{\prime}$ acides gras. La transformation de certains acides gras insaturés comme l'acide oléique (par ozonolyse) ou ricinoléique $(\mathrm{NaOH}$, $400{ }^{\circ} \mathrm{C}$ ) peut conduire à des intermédiaires spécifiques (nombre impair de carbones) et bifonctionnels. Des réactions de dimérisation peuvent également être envisagées pour obtenir des molécules à plus de 22 atomes de carbone.

\section{Les principaux agro-tensioactifs}

\section{Les esters de sorbitan ou mannitan}

Utilisés depuis des décennies, les esters de sorbitan ou de mannitan sont obtenus par estérification d'un polyol (sorbitol ou mannitol) en présence d'un acide gras ou coupe d'acides gras. $C$ sont des mono-, di-, tri- voire tétra-esters... Leurs HLB sont comprises entre 2 et 8 . Ces agrotensioactifs sont utilisés en cosmétique et en santé animale (vaccination).

\section{Les alkylpolyglucosides (APG)}

Les alkylpolyglucosides(APG) sont obtenus à partir de glucose et d'acides gras. Les APG commercialisés sont souvent 


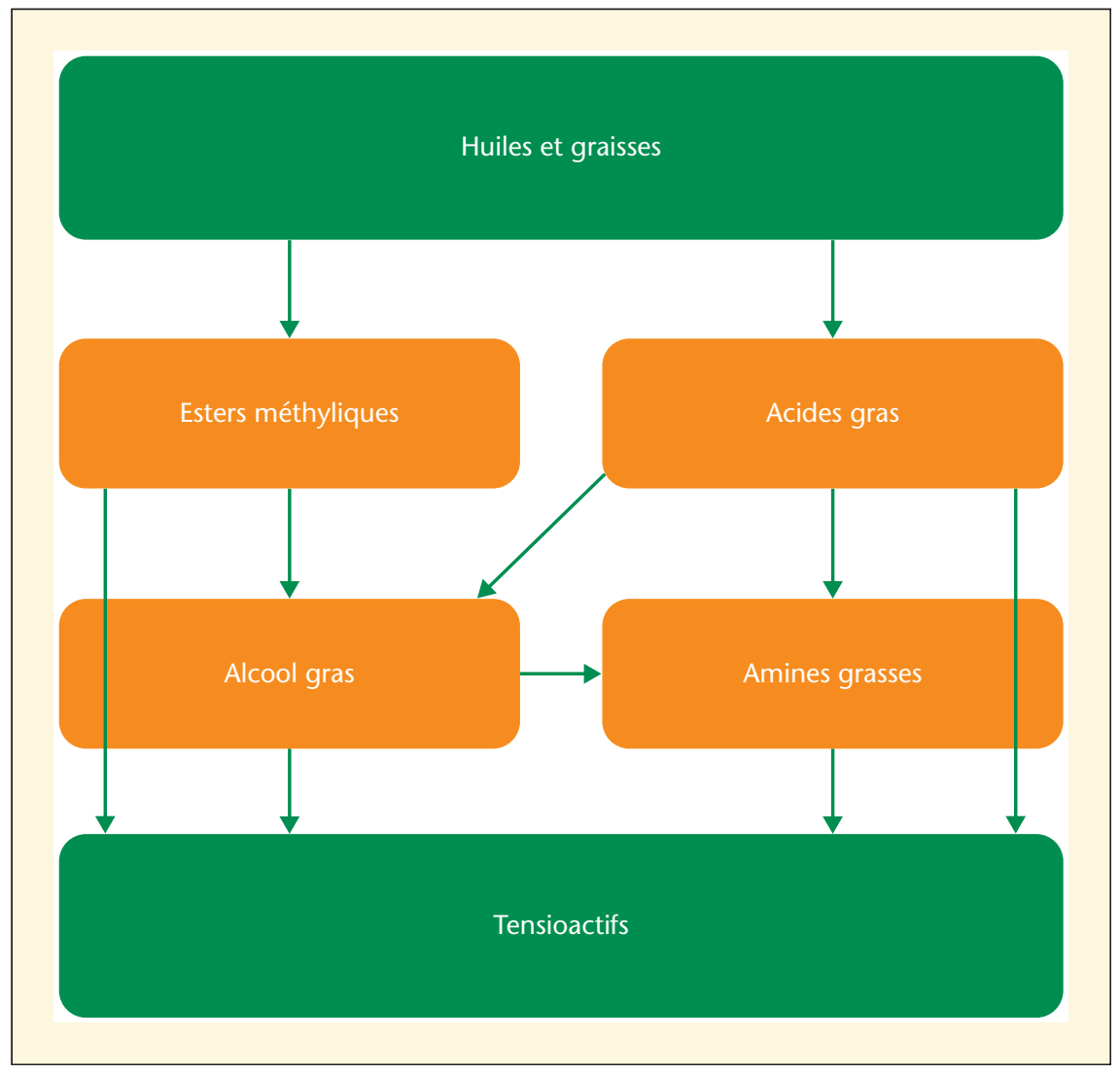

Figure 5. Les intermédiaires chimiques de base utilisés pour la synthèse de tensioactifs sont principalement les acides gras, les esters méthyliques, les amines grasses et les alcools gras.

des mélanges d'isomères dans lequels I'unité saccharidique varie de 1 à 6 . Les APG représentent un marché mondial de plus de $100000 \mathrm{t} / \mathrm{an}$. Ils sont utilisés en tant qu'agents moussants ou comme émulsifiants, cotensioactifs en détergence (produit vaisselle), tensioactifs doux pour les soins corporels (crèmes, gels douche), nettoyants et agents mouillants pour les produits phytosanitaires.

Les principaux producteurs sont Cognis, Sepic, BASF.

\section{Les sucro-esters}

Les sucro-esters sont produits par transestérification avec un sucre (le saccharose) et des esters éthyliques d'acides gras. Sans odeur et sans couleur, les sucro-esters sont utilisés dans le domaine de l'agro-alimentaire et de la cosmétique. Les sucro-esters sont des émulsifiants de type non-ionique, dont I'HLB dépend du degré d'estérification : selon les conditions opératoires, on obtient des mono-, di-, tri- ou polyesters de saccharose. Les mono- ou diesters saccharose sont utilisés des propriétés applicatives et environnementales proches des APG.

\section{Les alcools gras et esters gras éthoxylés}

Les alcools gras et esters gras éthoxylés sont préparés à partir d'alcool gras et d'oxyde d'éthylène ou de propylène. Ce sont d'excellents détergents, même à basse température. Ils peuvent également être utilisés dans la formulation de crèmes et dans l'industrie textile

\section{Gamme INUTEC ${ }^{\mathbb{R}}$ de Beneo Orafti}

Orafti a mis au point un agro-tensioactif à partir d'hydrolysats d'inuline (polymères de fructose) et de chaînes alkyles, sous la gamme INUTEC ${ }^{\circledR}$. Les applications sont diverses : cosmétiques, peintures vernis, produits pharmaceutiques.

\section{Les lipo-aminoacides}

Les lipo-aminoacides sont obtenus par acylation d'acides aminés par un chlorure d'acides (RCOCl). On peut citer PROTEOL ${ }^{\circledR}$ de Seppic valorisé en cosmétique : gels douche, shampoings. . .

\section{Les phospholipides}

Les phospholipides sont extraits des huiles (soja, colza, tournesol, œuf), et peuvent être utilisés directement sans transformation ultérieure. Les plus connus sont la phosphatidyl choline (lécithine) et la phophatidyl-éthanolamine. Les lécithines (E322) sont utilisées comme émulsifiants dans l'industrie alimentaire. Elles sont aujourd'hui majoritairement extraites du soja. On peut citer le distributeur Henry Franc.

\section{Les alpha sulfonyl-methyl-esters}

Les alpha sulfonyl-methyl-esters sont produits à partir d'esters méthyliques d'acides gras sulfonatés. $100 \%$ biodégradables, disposant d'un fort pouvoir détergent, ils peuvent substituer les $\mathrm{LABs}$ pour certaines applications : liquides vaisselles, poudres à laver le linge...

\section{RADIA ${ }^{\mathbb{R}}$-EASYSURF : une nouvelle génération de surfactants basés sur les pentoses (sucres à 5 carbones)}

La création de Wheatoleo, joint-venture à 50/50 entre le groupe français ARD 
(pour la R\&D) et le Belge Oleon (pour la production), a été officialisée au mois de mars 2007. L'activité de cette nouvelle société est consacrée aux surfactants verts issus de son de blé et utilisés comme tensioactifs (agréés Ecocert) dans la cosmétique.

Ces produits à base de polypentosides d'alkyle (APP) seront commercialisés conjointement par les équipes commerciales d'Oleon et Soliance, dans le monde entier.

ARD s'intéresse déjà depuis longtemps à la valorisation du son de blé, au travers notamment de l'extraction et de la purification des pentoses issus de la fraction hémicellulosique. La création de cette joint-venture va lui permettre de passer à la phase commerciale de ce travail.

\section{Nouveautés}

\section{SIMULGREEN 18-2 (Hydroxy stearyl glucoside) de la société Seppic}

Composé d'alcool hydroxystéarique issu d'huile de ricin et de glucose, le Simulgreen 18-2 est un émulsifiant H/E polyvalent, produit en accord avec les principes de chimie verte. Il est adapté à la fois pour les lotions et pour les crèmes, et prévient les phénomènes de savonnage à l'application.
RHODAPEX ESB-70 NAt Sodium

Laureth Sulfate d'origine végétale de Rhodia

Rhodapex ${ }^{\circledR}$ ESB-70 NAT est un tensioactif SLES (Sodium Laureth Sulfate) $\mathrm{d}^{\prime}$ origine végétale et renouvelable, idéal pour les formulations naturelles et de haute performance des shampoings, gels douche, savons liquides et produits d'entretien. Il est fabriqué à partir d'oxyde d'éthylène issu de canne à sucre et d'alcool laurique provenant d'huile de palme. Facilement biodégradable, il permet une réduction de $20 \%$ des émissions de gaz à effet de serre par rapport à son équivalent pétrochimique.

Pour plus d'informations consultez l'agrobiobase.com, la base de données des bioproduits.

\section{Les perspectives}

Les agro-tensioactifs, renouvelables, jouissent $d$ 'une très bonne image (faible toxicité et écotoxicité, une biodégradabilité supérieure aux tensioactifs d'origine pétrochimique, et une moindre agressivité sur la peau...), qui laisse penser que leur taux de pénétration devrait s'accroître dans les années à venir. Ils sont aujourd'hui positionnés sur des applications de niche et à haute valeur ajoutée. Les secteurs de la détergence resteront difficiles d'accès... La restriction à terme de substances telles que les éthoxylats, laisse entrevoir des marchés de plus grande consommation.

Le développement de nouveaux agrotensioactifs passe notamment par l'élargissement de la gamme des intermédiaires chimiques d'origine renouvelable: nouvelles sources de synthons originaux à travers le développement de nouvelles cultures (exemples: huile de cuphéa avec des acides gras à courtes chaînes, le colza érucique, les algues) et de nouvelles synthèses de synthons plus performantes.

\section{POUR EN SAVOIR PLUS}

Tensioactifs à base de substances renouvelables. Actualité chimique. Novembre-décembre 2002.

Borredon ME, Mouloungui Z. Chimie pour le développement durable - Les biotensioactifs - (Ppoint 02/10/06).

ADEME. Segmentation des utilisations des agro-tensioactifs et de leurs marchés potentiels. Marché actuel des bioproduits industriels et des biocarburants \& évolutions prévisibles à échéance 2015/2030 - synthèse Avril 2007. ALCIMED pour l'ADEME.

Tensioactifs et oléagineux : étude sur les matières premières oléagineuses disponibles sur le marché européen - catalogue des fournisseurs d'intermédiaires chimiques. ADEME - AGRICE.

Plateforme de veille et d'intelligence économique Tremplin - www.iar-pole.com. 